\title{
A non-invasive assessment of skin carotenoid status through reflection spectroscopy is a feasible, reliable and potentially valid measure of fruit and vegetable consumption in a diverse community sample
}

\author{
Stephanie Bell Jilcott Pitts ${ }^{1, *}$, Lisa Jahns ${ }^{2}$, Qiang Wu ${ }^{3}$, Nancy E Moran ${ }^{4}$, Ronny A Bell ${ }^{1}$, \\ Kimberly P Truesdale ${ }^{5}$ and Melissa N Laska ${ }^{6}$ \\ 'Department of Public Health, East Carolina University, 600 Moye Boulevard, Mailstop 660, Greenville, NC \\ 27834, USA: ${ }^{2}$ USDA/ARS Grand Forks Human Nutrition Research Center, Grand Forks, ND, USA: ${ }^{3}$ Department \\ of Biostatistics, East Carolina University, Greenville, NC, USA: ${ }^{4}$ USDA/ARS Children's Nutrition Research Center, \\ Department of Pediatrics, Baylor College of Medicine, Houston, TX, USA: ${ }^{5}$ Department of Nutrition, University of \\ North Carolina at Chapel Hill, Chapel Hill, NC, USA: ${ }^{6}$ Division of Epidemiology \& Community Health, University of \\ Minnesota, Minneapolis, MN, USA
}

Submitted 4 July 2017: Final revision received 21 November 2017: Accepted 21 November 2017: First published online 19 February 2018

\begin{abstract}
Objective: To assess the feasibility, reliability and validity of reflection spectroscopy (RS) to assess skin carotenoids in a racially diverse sample.

Design: Study 1 was a cross-sectional study of corner store customers ( $n 479$ ) who completed the National Cancer Institute Fruit and Vegetable Screener as well as RS measures. Feasibility was assessed by examining the time it took to complete three RS measures, reliability was assessed by examining the variation between three RS measures, and validity was examined by correlation with self-reported fruit and vegetable consumption. In Study 2, validity was assessed in a smaller sample ( $n$ 30) by examining associations between RS measures and dietary carotenoids, fruits and vegetables as calculated from a validated FFQ and plasma carotenoids. Setting: Eastern North Carolina, USA.

Results: It took on average 94.0 s to complete three RS readings per person. The average variation between three readings for each participant was $6.8 \%$. In Study 2 , in models adjusted for age, race and sex, there were statistically significant associations between RS measures and (i) FFQ-estimated carotenoid intake $(P<0 \cdot 0001)$; (ii) FFQ-estimated fruit and vegetable consumption $(P<0 \cdot 010)$; and (iii) plasma carotenoids $(P<0 \cdot 0001)$.

Conclusions: RS is a potentially improved method to approximate fruit and vegetable consumption among diverse participants. RS is portable and easy to use in field-based public health nutrition settings. More research is needed to investigate validity and sensitivity in diverse populations.
\end{abstract}

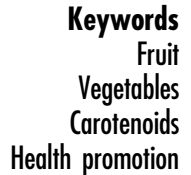

A diet rich in fruits and vegetables is associated with lower rates of obesity ${ }^{(1-3)}$, cancer $^{(4)}, \mathrm{CVD}^{(5,6)}$, diabetes ${ }^{(7)}$ and allcause mortality ${ }^{(8,9)}$. However, in general, the US population does not consume the recommended amounts of fruits and vegetables ${ }^{(10)}$. This is especially true for rural ${ }^{(11)}$ and disadvantaged $^{(12)}$ populations. Thus, examining and addressing the low consumption of fruits and vegetables, particularly in disadvantaged populations, is important to prevent chronic disease. To effectively design and test interventions to increase fruit and vegetable consumption in these populations, valid and reliable measures are needed. Traditional methods of self-reported dietary assessment have limitations due to recall and social desirability biases $^{(13,14)}$, and even greater limitations in underserved populations due to low literacy and numeracy ${ }^{(15)}$. The current reference measure for fruit and vegetable consumption, 
blood carotenoid concentration, is an expensive, timeintensive and invasive measurement ${ }^{(16)}$. Thus, a critical gap in assessing the effectiveness of community- and policy-level interventions to promote fruit and vegetable consumption is the lack of a non-invasive, easy-to-use, valid and reliable measure of fruit and vegetable consumption in underserved populations.

Skin carotenoid status assessed by resonance Raman spectroscopy has emerged as a promising new biomarker of fruit and vegetable intake ${ }^{(17)}$. A wide distribution of skin carotenoid levels has been demonstrated ${ }^{(18,19)}$ with high reproducibility over 6 months ${ }^{(19,20)}$ and validity compared with plasma, skin biopsy and reported fruit and vegetable intake $^{(19)}$, all necessary characteristics for a biomarker of nutritional status and food intake. Reflection spectroscopy (RS) is a potentially improved method of measuring skin carotenoid status ${ }^{(21)}$. However, to date, RS validation studies have been conducted in a laboratory setting and primarily in white populations ${ }^{(21,22)}$. Additionally, RS has not been tested in terms of feasibility, reliability and validity in a community-based field setting among racially and ethnically diverse individuals. Therefore, the present study's purpose was to assess the feasibility, reliability and validity of RS in assessing skin carotenoids in a racially diverse community sample recruited from a field-based food retail venue (small corner stores); moreover, to examine validity in greater detail among a purposefully selected group of African-American and Caucasian participants, we examined associations between RS-assessed skin carotenoids, plasma carotenoids and dietary data among participants recruited from a medical school listserv.

\section{Methods and materials}

\section{Setting and participants}

\section{Study 1}

This pilot study was part of a larger evaluation project designed to assess the effect of the North Carolina Healthy Food Small Retailer Program, a programme funded by the NC Legislature to improve the offerings of healthy foods in small corner stores in Eastern North Carolina, on dietary behaviours among rural and disadvantaged residents of Eastern North Carolina, USA ${ }^{(23)}$. As part of our evaluation project, in February-April 2017 we pilot-tested a customer intercept survey and the RS device (described below) among 466 customers in sixteen Eastern North Carolina small convenience/corner stores. Participants were eligible if they were over 18 years of age, a store customer and spoke English. Participants were given an information sheet describing the study and informed consent by signature was not required.

\section{Study 2}

In addition, to examine associations between plasma carotenoids and RS-assessed carotenoids in people self-identifying as African American ( $n$ 17) and nonHispanic White ( $n$ 13), in July-August 2017 we recruited and enrolled thirty participants from a medical school email listserv. Participants were screened for eligibility, which included not smoking, BMI between 18.5 and $29.9 \mathrm{~kg} / \mathrm{m}^{2}$, healthy/no history of chronic disease, and not participating in any weight-loss or other lifestyle intervention programmes. After being screened and determined eligible, participants were invited to the East Carolina Diabetes and Obesity Institute lab in a fasting state. At the Institute, they read the informed consent materials, had the opportunity to ask questions and provided a signature signifying their informed consent. Participants had their blood drawn; had weight, height and skin carotenoids (RS-assessed) measured using standard procedures; and then completed an FFQ and Demographic Questionnaire (measures are described in detail below).

\section{Self-reported fruit and vegetable consumption}

Study 1

Corner store participants self-reported consumption of fruits and vegetables over the past month using the National Cancer Institute (NCI) 'all day' Fruit and Vegetable Screener (F\&V Screener) ${ }^{(23)}$ (scoring algorithms are located at http:// www.epi.grants.cancer.gov/diet/screeners/fruitveg/scoring/ allday.html\#how). Frequency response options range from never, 1-3 times last month, 1-2 times per week, up to 5 or more times per day. The NCI F\&V Screener queries the frequency and amount for the following items: $100 \%$ Juice (orange, apple, grape, or grapefruit); Fruit (fresh, canned, frozen, no juice); Lettuce Salad; French Fries/Fried Potatoes; Other White Potatoes (baked, boiled and mashed potatoes, potato salad and white potatoes that are not fried); Cooked Dried Beans; Other Vegetables; and Tomato Sauce (tomato sauce on pasta or macaroni, rice, pizza and other dishes). The F\&V Screener also includes frequency for Vegetable Mixtures (foods such as sandwiches, casseroles, stews, stir-fry, omelettes and tacos). Daily servings of fruits and vegetables were calculated using the standard algorithm (that includes frequency and amount consumed) as provided on the NCI website ${ }^{(24)}$. While the F\&V screener does not gather detailed information on specific carotenoidrich fruits and vegetables servings consumed, it is a preferred tool for use in community-based nutrition studies because of its simplicity and brevity of administration and analysis $^{(24,25)}$. For this pilot analysis, our goal was to determine if the RS device (a rapid assessment tool) agreed with the F\&V Screener (another rapid assessment tool) to assess fruit and vegetable consumption.

Study 2

For the smaller validation follow-up study, our objective was to determine if intakes of specific fruits and vegetables or of total dietary carotenoids were correlated with RS device measures in a diverse population. For this study, 
the Fred Hutchinson Cancer Research Center (FHCRC) FFQ was used to determine detailed information about dietary carotenoid intake and fruit and vegetable consumption. The self-administered FFQ asks participants to report the frequency and portion size of 125 food and beverage line items over a defined time period (1 month in our study) ${ }^{(26,27)}$. Dietary carotenoids and daily fruit and vegetable servings were calculated using the FHCRC standard algorithms ${ }^{(26,27)}$.

\section{Skin carotenoid assessment}

In addition to self-reported dietary assessment, we also used the RS device to evaluate the use of skin carotenoids as a non-invasive, objective measure of dietary intake of fruits and vegetables. The RS device (Veggie Meter; Longevity Link Corp., Salt Lake City, UT, USA) was developed by those who originally developed resonance Raman spectroscopy as an objective indicator of carotenoid status $^{(17,21,22)}$. RS involves measurement of the skin carotenoid absorption via reflection under application of topical pressure $^{(21)}$. Applying a modest amount of topical pressure allows the carotenoid absorption spectra to be derived from the reflection spectra; the topical pressure provides an increase in the contrast between carotenoid absorption strength and the combined absorption background from other chromophores ${ }^{(21)}$.

Trained research staff directed participants to place the right or left index finger (the side most comfortable for the participant) on a lens on the top of the RS device. A lever was then lowered over the finger which applied a gentle pressure, which temporarily blanches the skin by restricting blood flow, as is necessary to minimize interference of blood $\mathrm{Hb}$ with the RS carotenoid measurements. The device is linked to a tablet computer and shows the participant where his or her skin carotenoid reading falls on a histogram of all other readings prior to their measure. Each participant's index finger was scanned three times and the average of the last two readings was used in this analysis. When time permitted, data collectors recorded the time three readings took using a stopwatch and recorded any qualitative comments made by participants specific to the RS device.

\section{Plasma carotenoid measurements}

Plasma was isolated by centrifugation in EDTA-containing Vacutainer tubes. Plasma was aliquoted and stored at $-80^{\circ} \mathrm{C}$ and shipped on dry ice to the USDA/ARS Grand Forks Human Nutrition Research Center analytical lab. Human plasma carotenoid analysis was performed by LC-MS/MS. In brief, under reduced lighting, $10 \mu \mathrm{l}$ plasma is mixed with $990 \mu \mathrm{l}$ of methanol containing internal standard, centrifuged to remove protein, and $25 \mu \mathrm{l}$ of the supernatant is injected on to a C-30 column (YMC Carotenoid). The gradient elution method was previously described $^{(28)}$. Extraction efficiency was adjusted relative to the internal standard and analytes were quantitated by external calibration curves.

\section{Covariates}

Covariates thought to possibly influence self-reported fruit and vegetable consumption as well as carotenoid absorption and biodistribution ${ }^{(19,29-31)}$ were queried and included in predictive models.

\section{Study 1}

On the customer intercept survey, we asked participants to self-report race/ethnicity, smoking status (yes/no), age (continuous in years), sex (male, female), and height and weight (to calculate BMI). Response options for race/ethnicity included American Indian or Alaskan Native, Asian, Black or African American, Native Hawaiian or Pacific Islander, and White; and for ethnicity Hispanic or Latino or non-Hispanic or Latino, or Other/Refused. We dichotomized race/ethnicity as African American and non-African American to maximize power in analyses.

\section{Study 2}

Participants were asked to self-report race/ethnicity, age (continuous in years), sex (male, female, other), income and education levels.

\section{Data analysis}

\section{Study 1}

We assessed feasibility of the RS device by examining the time it took to obtain the three readings and anecdotal comments made by customers, as well as the ease of setting up and using the RS device in a small convenience/corner store. We examined reliability using the $\mathrm{CV}$ of the triplicate measure (also of the second two measures, assuming the first measure was a 'practice run'), calculated as the standard deviation of the three (or two) readings divided by their mean. We examined whether RS scores were normally distributed using the Kolmogorov-Smirnov test. We assessed agreement between RS scores and the easy-toimplement NCI F\&V Screener tool (convergent validity) by using linear regression to examine associations between RS scores and self-reported NCI fruit and vegetable daily servings, controlling for race, smoking status, age and sex.

\section{Study 2}

We examined associations between RS measures and (i) intake of total carotenoids and servings of fruits and vegetables as calculated from the FHCRC FFQ and (ii) plasma carotenoids as determined from blood drawn in a fasting state, using Pearson's correlation coefficients and linear regression models, adjusted for race, age and sex. (All participants were non-smokers.) The statistical software package SAS version 9.4 was used for all analyses. 


\section{Results}

Table 1 includes participant (corner store customer) characteristics and indicates that the majority (65.9\%) were African American and $42.7 \%$ had an annual household income of less than \$US 25000 . The mean age was 43.3 years, mean daily servings of fruits and vegetables were 1.8 and $2 \cdot 0$, respectively, and mean BMI was $30 \cdot 0 \mathrm{~kg} / \mathrm{m}^{2}$. The mean RS score (for three readings) was 234 RS intensities.

We recorded the time for the process of scanning the finger three times using the RS device for 151 of the total of

Table 1 Characteristics of 479 corner store customers (Study 1), Eastern North Carolina, USA, February-April 2017

\begin{tabular}{|c|c|c|}
\hline Characteristic & Frequency & $\%$ \\
\hline \multicolumn{3}{|l|}{ Race/ethnicity } \\
\hline African American & 309 & 65.9 \\
\hline Non-African American* & 160 & $34 \cdot 1$ \\
\hline Missing or refused & 10 & $2 \cdot 1$ \\
\hline \multicolumn{3}{|l|}{ Annual household income (\$US) } \\
\hline$<25000$ & 176 & $42 \cdot 7$ \\
\hline $25000-50000$ & 146 & 35.4 \\
\hline$>50000$ & 90 & 21.9 \\
\hline Missing & 67 & $14 \cdot 0$ \\
\hline \multicolumn{3}{|l|}{ Sex } \\
\hline Female & 196 & 41.2 \\
\hline Male & 280 & 58.8 \\
\hline Missing & 3 & 1.0 \\
\hline \multicolumn{3}{|l|}{ Smoking status } \\
\hline Yes & 228 & $47 \cdot 6$ \\
\hline \multirow[t]{2}{*}{ No } & 251 & $52 \cdot 4$ \\
\hline & Mean & SD \\
\hline Age (years) & $43 \cdot 3$ & $15 \cdot 1$ \\
\hline BMI $\left(\mathrm{kg} / \mathrm{m}^{2}\right)$ & $30 \cdot 0$ & 7.9 \\
\hline RS score (mean of three readings) & 234.2 & $86 \cdot 2$ \\
\hline RS score (mean of the last two readings) & 235.5 & 87.5 \\
\hline Daily servings of fruit (NCI F\&V Screener) & 1.8 & $2 \cdot 2$ \\
\hline Daily servings of vegetables (NCI F\&V Screener) & $2 \cdot 0$ & 1.9 \\
\hline $\begin{array}{l}\text { Daily servings of fruits and vegetables (NCI F\&V } \\
\text { Screener) }\end{array}$ & $3 \cdot 8$ & $3 \cdot 2$ \\
\hline
\end{tabular}

RS, reflection spectroscopy; NCI, National Cancer Institute; F\&V Screener, 'all day' Fruit and Vegetable Screener.

*The non-African American group included five American Indians/Alaska Natives, one Asian, fifteen Hispanics/Latinos, 126 Whites and thirteen Other racial groups. Nine were missing and one refused.
479 participants. On average, this process took 94.0 (sD 27.7) s. The RS device was generally received well by participants. Responses included positive feedback, including: 'This is interesting, this is cool stuff'; 'I want one of these. That's cool'. Some participants wanted to know more about what the score meant, and tried to relate the RS score to actual fruit and vegetable consumption, such as: 'I know my score is high because I eat a lot of fruits and vegetables'; 'My score is low - I need to eat more fruits and vegetables'. Not all responses were positive and some indicated hesitance, or a desire to get the readings done more quickly: 'Why are y'all doing this?'; 'Why do the numbers change?'

Intra-individual variation of the triplicate measurements was $6.8 \%$. When the second two of the three readings were used, the variation of these two readings was 5.6\%. Table 2 shows that among African American participants, the variation was $6.8 \%$ for the triplicate measurements and $5.6 \%$ for the second two readings. Among the non-African American participants, the variation was 6.7 and $5.7 \%$ for three and two readings, respectively.

\section{Study 1}

We examined associations between self-reported daily servings of fruits and vegetables (NCI F\&V Screener) and RS scores, adjusted for race, smoking, age, sex and BMI. There were no statistically significant associations between RS scores and NCI-assessed daily servings of fruits and vegetables (see Table 3 ). The $R^{2}$ was 0.08 for each of the three models. When we conducted a sensitivity analysis, examining only African American and White participants, the results did not change.

\section{Study 2}

For the small validation study, mean age of participants was 32.9 (SD 11.8) years, mean BMI was 25.1 (SD 2.7) kg/m², mean skin carotenoid score was 296.4 (SD 110.3) RS intensities and mean carotenoids from food was 16954.6 (SD 12280.9 ) $\mu \mathrm{g} / \mathrm{d}$. Seven (24\%) reported an annual household income less than \$US 19999 and nineteen (65\%) reported having a college education or greater

Table 2 Mean and standard deviation of age, BMI, reflection spectroscopy (RS) scores, CV of RS scores, and self-reported consumption of fruits and vegetables as reported on the National Cancer Institute (NCl) Fruit and Vegetable Screener (F\&V Screener), stratified by African American v. non-African American (Study 1), Eastern North Carolina, USA, February-April 2017

\begin{tabular}{|c|c|c|c|c|}
\hline & \multicolumn{2}{|c|}{$\begin{array}{l}\text { African Americans } \\
\text { (n 309) }\end{array}$} & \multicolumn{2}{|c|}{$\begin{array}{l}\text { Non-African Americans } \\
(n 160)\end{array}$} \\
\hline & Mean & SD & Mean & SD \\
\hline Age (years) & $44 \cdot 6$ & $14 \cdot 2$ & $40 \cdot 6$ & $16 \cdot 4$ \\
\hline $\mathrm{BMI}\left(\mathrm{kg} / \mathrm{m}^{2}\right)$ & 30.5 & $8 \cdot 4$ & $29 \cdot 0$ & 6.7 \\
\hline RS score (mean of three readings) & $239 \cdot 0$ & $85 \cdot 8$ & $225 \cdot 4$ & $87 \cdot 8$ \\
\hline RS score (mean of two readings) & $241 \cdot 1$ & $87 \cdot 5$ & 225.4 & $88 \cdot 1$ \\
\hline CV for the RS device (three readings) & $6 \cdot 8$ & $8 \cdot 2$ & $6 \cdot 7$ & $8 \cdot 1$ \\
\hline CV for the RS device (two readings) & $5 \cdot 6$ & 8.3 & $5 \cdot 7$ & $11 \cdot 1$ \\
\hline Daily servings of fruit (NCI F\&V Screener) & 1.8 & $2 \cdot 3$ & $2 \cdot 1$ & $2 \cdot 2$ \\
\hline Daily servings of vegetables (NCl F\&V Screener) & 1.9 & $1 \cdot 8$ & $2 \cdot 2$ & $2 \cdot 2$ \\
\hline
\end{tabular}


Table 3 Adjusted analyses examining associations between reflection spectroscopy (RS) scores for participants in Study 1 ( $n$ 479) and National Cancer Institute (NCl) Fruit and Vegetable Screener (F\&V Screener)-assessed fruits and vegetables (adjusted for age, race, sex, smoking) and between RS scores for participants in Study $2(n 30)$ and Fred Hutchinson Cancer Research Center (FHCRC) FFQ data and plasma carotenoids (adjusted for age, race and sex), Eastern North Carolina, USA, February-April 2017

\begin{tabular}{lcccc}
\hline Independent variable & Estimate & SE & $P$ value & $R^{2}$ \\
\hline NCI F\&V Screener - fruit (Study 1) & -1.34 & 1.85 & 0.471 & 0.08 \\
NCI F\&V Screener - vegetables (Study 1) & 1.88 & 2.09 & 0.370 & 0.08 \\
NCI F\&V Screener - fruit and vegetables (Study 1) & 0.04 & 1.25 & 0.975 & 0.08 \\
FHCRC FFQ - fruit and vegetables (Study 2) & 31.60 & 11.27 & 0.010 & 0.27 \\
FHCRC-FFQ - carotenoids (mg) (Study 2) & 0.007 & 0.001 & $<0.0001$ & 0.59 \\
Plasma carotenoids (nmol/l) (Study 2) & 0.07 & 0.01 & $<0.0001$ & 0.55 \\
\hline
\end{tabular}

Table 4 Associations (Pearson's correlation coefficient and $P$ values) between reflection spectroscopy (RS)-assessed skin carotenoids and self-reported fruit and vegetable consumption (daily servings), carotenoids from foods and plasma carotenoids, overall and stratified by African American v. non-African American (Study 2), Eastern North Carolina, USA, February-April 2017

\begin{tabular}{|c|c|c|c|c|c|c|}
\hline Group & $\begin{array}{l}\text { Total fruit and vegetable } \\
\text { consumption (daily servings) }\end{array}$ & $P$ value & $\begin{array}{l}\text { Total carotenoid intake } \\
\text { from food }(\mu \mathrm{g} / \mathrm{d})\end{array}$ & $P$ value & $\begin{array}{c}\text { Total plasma } \\
\text { carotenoids }(\mu \mathrm{mol} / \mathrm{l})\end{array}$ & $P$ value \\
\hline All $(n 30)$ & 0.48 & 0.009 & 0.69 & $<0.0001$ & 0.71 & $<0.0001$ \\
\hline African American ( $n$ 17) & 0.12 & 0.635 & 0.70 & 0.002 & 0.54 & 0.024 \\
\hline White ( $n$ 13) & 0.84 & 0.001 & 0.75 & 0.003 & 0.87 & $<0.0001$ \\
\hline
\end{tabular}

(data not shown). Table 4 shows correlations between FFQ-assessed carotenoids and fruits and vegetables, plasma carotenoids and skin carotenoids as assessed using RS, for the total sample ( $n$ 30) and stratified by race. There were significant correlations for all associations (ranging from $r=0.48$ to $r=0.86$ ) except for the association between FFQ-assessed fruits and vegetables and RS among African Americans. Table 3 shows that in models adjusted for age, race and sex, there were statistically significant associations between RS measures and (i) FFQ-estimated carotenoid intake $(P<0 \cdot 0001)$; (ii) FFQ-estimated fruit and vegetable consumption $(P<0 \cdot 010)$; and (iii) plasma carotenoids $(P<0.0001)$.

\section{Discussion}

We found that the RS device was a feasible, reliable and potentially valid assessment tool for estimating carotenoidcontaining fruit and vegetable consumption in this racially diverse sample. The device could be set up in a small corner store, in areas such as a fountain beverage counter or small table. It took, on average, $94 \mathrm{~s}$ to complete three readings in each participant, making it feasible for an intercept survey in a variety of community settings. In addition, in field-based community settings where timeefficient data collection is needed, two readings from the RS device should be adequate to evaluate and assess change.

Some participants were interested to know what the numbers meant, indicating that in future studies using the RS device a simple explanation should be used by data collectors, such as: 'The number is a measure of the level of carotenoids, or the colourful substances in some fruits and vegetables, in your skin'. Our data collectors usually referred customers to the histogram on the laptop and told individuals that their line on the histogram represented where their values fell in relation to all the values ever taken by this machine.

The RS device had $6.8 \%$ variation in the triplicate measure in our field-based study, which was not substantively different between participants of different racial groups. Furthermore, in adjusted analyses, we found positive associations between self-reported fruit and vegetable consumption and RS scores in Study 2, indicating that the RS device is potentially valid for estimating fruit and vegetable consumption in a diverse community sample.

In Study 1, we did not find that NCI-assessed fruits and vegetables were associated with RS-assessed skin carotenoids; yet in Study 2, we found that FHCRC FFQ-assessed fruits and vegetables and carotenoids from foods were positively associated with RS-assessed skin carotenoids. This is perhaps due to the NCI F\&V Screener being a validated tool for rapid assessment of total fruit and vegetable consumption $v$. carotenoid-rich fruit and vegetable consumption. In addition, it is likely that our population consumed a large proportion of potatoes and French fries, which are detected by the NCI screener but do not contain carotenoids. Study 2 results indicate that the RS device is a useful tool to assess non-potato and non-juice fruit and vegetable intake, which are the fruits and vegetables that are encouraged by the Dietary Guidelines for Americans. However, we did find that the association between self-reported total fruits and vegetables and RS-assessed skin carotenoids was not significant among African Americans, suggesting that there could be differences in genetics ${ }^{(31)}$ or dietary patterns that account 
for these differences. Our combined results from Studies 1 and 2 demonstrate the need to delve more deeply into understanding the RS device and associations with fruit and consumption given differences in race/ethnicity, ancestry and genetics, and regional food patterns, to further ready the RS device as a tool for nutrition surveillance and monitoring as well as evaluation of public health nutrition interventions.

The main limitation of the present study was the small sample size for our validation study examining correlations between RS scores and plasma carotenoids. This should be replicated in larger samples. In addition, nicotine could stain the skin of smokers, as could the powder from chilli peppers found on potato chips and other processed foods ${ }^{(32)}$, and this could potentially affect RS readings.

In the current study we found that the RS device holds promise for evaluation of community- and policy-level nutrition interventions in a variety of community settings. Further field validation is needed to examine associations between the RS device and the reference standard of blood carotenoids in diverse racial and ethnic groups and in a variety of community settings.

\section{Acknowledgements}

Acknowledgements: The authors would like to thank Dr Werner Gellermann of Longevity Link Corp. for initial loan of the Veggie Meter and for providing expertise and guidance on this paper. They also gratefully acknowledge the data collection efforts of Taras Grinchak, Trevor Hamlett, Cameron Howell, Archana Kaur and Mary Jane Lyonnais, as well as all East Carolina Diabetes and Obesity Institute staff and study participants and corner store owners and managers. Financial support: This study was supported by the US Department of Agriculture (Duke-UNC Behavioral Economics and Choice Research Center New Perspectives Fellowship, grant number 59-5000-4-0062); the Brody Brothers Endowment Fund; and the East Carolina University Departments of Public Health and Biostatistics. This work was also supported in part by the US Department of Agriculture (grant numbers 59-5000-4-0062, 3062-51000-051-00D and 3092-51000056-036, N.E.M.); and by the National Institutes of Health National Center for Complementary and Integrative Health and Office of Dietary Supplements (grant number R00 AT008576, N.E.M.). The content is solely the responsibility of the authors and does not necessarily represent the official views of the National Institutes of Health or the US Department of Agriculture. Conflict of interest: None. Authorship: S.B.J.P., L.J., N.E.M. and M.N.L. designed the study. S.B.J.P., L.J., R.A.B., K.P.T., N.E.M. and M.N.L. guided design of data analysis and interpretation. Q.W. analysed the data. All authors assisted with drafting and revising the manuscript for important intellectual content. All read and approved the final submitted manuscript.
All authors agree to be accountable for all aspects of the work. Ethics of human subject participation: This study was reviewed and approved by the East Carolina University Institutional Review Board (IRB \#16-002420).

\section{References}

1. Bertoia ML, Mukamal KJ, Cahill LE et al. (2015) Changes in intake of fruits and vegetables and weight change in United States men and women followed for up to 24 years: analysis from three prospective cohort studies. PLoS Med 12, e1001878.

2. Gibbs BB, Kinzel LS, Gabriel KP et al. (2012) Short- and long-term eating habit modification predicts weight change in overweight, postmenopausal women: results from the WOMAN study. J Acad Nutr Diet 112, 1347-1355.e2.

3. Ledoux T, Hingle MD \& Baranowski T (2011) Relationship of fruit and vegetable intake with adiposity: a systematic review. Obes Rev 12, e143-e150.

4. Bradbury KE, Appleby PN \& Key TJ (2014) Fruit, vegetable, and fiber intake in relation to cancer risk: findings from the European Prospective Investigation into Cancer and Nutrition (EPIC). Am J Clin Nutr 100, Suppl. 1, 394S-398S.

5. Griep LMO, Verschuren WM, Kromhout D et al. (2011) Colours of fruit and vegetables and 10-year incidence of CHD. Br J Nutr 106, 1562-1569.

6. He FJ, Nowson CA \& MacGregor GA (2006) Fruit and vegetable consumption and stroke: meta-analysis of cohort studies. Lancet 367, 320-326.

7. Carter P, Gray LJ, Troughton J et al. (2010) Fruit and vegetable intake and incidence of type 2 diabetes mellitus: systematic review and meta-analysis. BMJ 341, c4229.

8. Wang X, Ouyang Y, Liu J et al. (2014) Fruit and vegetable consumption and mortality from all causes, cardiovascular disease, and cancer: systematic review and dose-response meta-analysis of prospective cohort studies. BMJ 349, g4490.

9. Nguyen B, Bauman A, Gale J et al. (2016) Fruit and vegetable consumption and all-cause mortality: evidence from a large Australian cohort study. Int J Behav Nutr Phys Act 13, 9.

10. Krebs-Smith SM, Guenther PM, Subar AF et al. (2010) Americans do not meet federal dietary recommendations. J Nutr 140, 1832-1838.

11. Lutfiyya MN, Chang LF \& Lipsky MS (2012) A cross-sectional study of US rural adults' consumption of fruits and vegetables: do they consume at least five servings daily? BMC Public Health 12, 280.

12. Kamphuis CB, Giskes K, de Bruijn G et al. (2006) Environmental determinants of fruit and vegetable consumption among adults: a systematic review. BrJ Nutr 96, 620-635.

13. Schoeller DA, Thomas D, Archer E et al. (2013) Self-reportbased estimates of energy intake offer an inadequate basis for scientific conclusions. Am J Clin Nutr 97, 1413-1415.

14. Dhurandhar NV, Schoeller D, Brown AW et al. (2015) Energy balance measurement: when something is not better than nothing. Int J Obes (Lond) 39, 1109-1113.

15. Thompson FE, Subar AF, Loria CM et al. (2010) Need for technological innovation in dietary assessment. $J$ Am Diet Assoc 110, 48-51.

16. Institute of Medicine, National Academy of Sciences, Food and Nutrition Board, Panel on Dietary Antioxidants and Related Compounds (2000) Dietary Reference Intakes for Vitamin C, Vitamin E, Selenium, and Carotenoids. Washington, DC: National Academies Press.

17. Mayne ST, Cartmel B, Scarmo S et al. (2013) Resonance Raman spectroscopic evaluation of skin carotenoids as a biomarker of carotenoid status for human studies. Arch Biochem Biophys 539, 163-170. 
18. Scarmo S, Henebery K, Peracchio H et al. (2012) Skin carotenoid status measured by resonance Raman spectroscopy as a biomarker of fruit and vegetable intake in preschool children. Eur J Clin Nutr 66, 555-560.

19. Mayne ST, Cartmel B, Scarmo S et al. (2010) Noninvasive assessment of dermal carotenoids as a biomarker of fruit and vegetable intake. Am J Clin Nutr $\mathbf{9 2}$, 794-800.

20. Scarmo S, Cartmel B, Lin H et al. (2013) Single $v$. multiple measures of skin carotenoids by resonance Raman spectroscopy as a biomarker of usual carotenoid status. Br J Nutr 110, 911-917.

21. Ermakov IV \& Gellermann W (2012) Dermal carotenoid measurements via pressure mediated reflection spectroscopy. J Biophotonics 5, 559-570.

22. Ermakov IV, Whigham LD, Redelfs AH et al. (2016) Skin carotenoids as biomarker for vegetable and fruit intake: validation of the reflection-spectroscopy based 'Veggie Meter'. FASEB J 30, Suppl. 1, 409.3.

23. Jilcott Pitts SB, Wu Q, Truesdale KP et al. (2017) Baseline assessment of a healthy corner store initiative: associations between food store environments, shopping patterns, customer purchases, and dietary intake in Eastern North Carolina. Int J Environ Res Public Health 14, E1189.

24. Greene GW, Resnicow K, Thompson FE et al. (2008) Correspondence of the NCI fruit and vegetable screener to repeat $24-\mathrm{H}$ recalls and serum carotenoids in behavioral intervention trials. J Nutr 138, issue 1, 200S-204S.
25. Thompson FE, Subar AF, Smith AF et al. (2002) Fruit and vegetable assessment: performance of 2 new short instruments and a food frequency questionnaire. J Am Diet Assoc 102, 1764-1772.

26. Kristal AR, Vizenor NC, Patterson RE et al. (2000) Precision and bias of food frequency-based measures of fruit and vegetable intakes. Cancer Epidemiol Biomarkers Prev 9 , 939-944.

27. Kristal AR, Beresford SA \& Lazovich D (1994) Assessing change in diet-intervention research. Am J Clin Nutr 59, Suppl. 1, 185S-189S.

28. Melendez-Martinez AJ, Stinco CM, Liu C et al. (2013) A simple HPLC method for the comprehensive analysis of cis/trans $(Z / E)$ geometrical isomers of carotenoids for nutritional studies. Food Chem 138, 1341-1350.

29. Jahns L, Johnson LK, Mayne ST et al. (2014) Skin and plasma carotenoid response to a provided intervention diet high in vegetables and fruit: uptake and depletion kinetics. Am J Clin Nutr 100, 930-937.

30. Ermakov IV \& Gellermann W (2010) Validation model for Raman based skin carotenoid detection. Arch Biochem Biophys 504, 40-49.

31. Borel P (2012) Genetic variations involved in interindividual variability in carotenoid status. Mol Nutr Food Res 56, 228-240.

32. Whigham LD \& Redelfs AH (2016) Inter-finger variability and other influencing factors for using reflectance spectroscopy for skin carotenoid detection. FASEB J 30, Suppl. 1, 409.2. 